\title{
Survival of trauma patients who have prehospital tracheal intubation without anaesthesia or muscle relaxants: observational study
}

\author{
David Lockey, Gareth Davies, Tim Coats
}

In the United Kingdom, it is current practice for paramedics to perform tracheal intubation on trauma patients when the airway is compromised and basic airway manoeuvres have failed. Paramedics in Britain never use anaesthetic drugs or muscle relaxants to achieve intubation.

Anecdotal experience shows that patients who can be intubated without the use of drugs have a poor prognosis. We investigated mortality in a population of trauma patients who were intubated before reaching hospital without anaesthetic drugs being used.

\section{Participants, methods, and results}

We looked retrospectively at the database of a helicopter emergency medical service staffed by doctors and paramedics that is specifically targeted at trauma patients in a mainly urban area. We identified patients who had been intubated without drugs by paramedics or doctors, and we recorded whether they survived to hospital discharge. All patients were attended by physicians, but many of the patients were intubated by ground crew paramedics before the medical team arrived. Patients were taken to the nearest appropriate hospital by ground or air.

In a six year period, from January 1990 to December 1996, 1623 patients were intubated outside hospital. Of these, we excluded $143(8.8 \%)$ because they were not trauma patients. Of the remaining 1480 patients, $492(33.2 \%)$ were intubated without drugs: 275 (55.8\%) by physicians and $216(43.9 \%)$ by paramedics. Data regarding survival were not available for six of these patients, but of the remaining 486 patients, one (0.2\%) survived. This person had a cardiac arrest after penetrating chest trauma and underwent a thoracotomy on scene to relieve pericardial tamponade and suture the myocardium.

\section{Comment}

As almost all the trauma patients intubated without the use of drugs died, the value of this practice is doubtful. To allow easy passage of a tracheal tube without anaesthetic drugs, a patient must be profoundly unconscious, and a high likelihood of death might be expected. Despite this expectation, it was surprising that the outcome was almost always fatal.

Paramedics in Britain have been intubating without the use of anaesthetic drugs for more than 20 years, and many resources have been invested in teaching the skill. This intervention was introduced mainly to improve outcome following medical (non-traumatic) cardiac arrest, but recently the effectiveness of intubation in this situation has been questioned. ${ }^{1}$ In patients with severe trauma, airway compromise is a cause of prehospital death that can be prevented ${ }^{2}$ and simple airway manoeuvres can clear the airway to provide vital oxygenation.
Laryngoscopy and attempted intubation without drugs have potential risks, such as an increase in intracranial pressure, vomiting, and unrecognised oesophageal intubation. ${ }^{3}$ Some trauma systems use drugs to facilitate prehospital intubation-this is standard practice for paramedics and nurses in parts of the United States-but even if drugs are used, failed intubation rates can be high. ${ }^{4}$

There is little evidence about the optimum prehospital management of severe blunt injury, and there are no controlled trials of the different methods of airway management. As the role of the UK paramedic is under discussion $^{5}$ and there are few data from the United Kingdom about the rate of failed prehospital intubation in trauma patients, the use of non-drug assisted intubation deserves further scrutiny.

Contributors: DL, GD, and TC conceived the idea for the study DL collected the data and wrote the paper. The paper was discussed, revised, and edited by DL, GD, and TC. DL is the guarantor of the paper.

Funding: None declared.

Competing interests: None declared.

1 Guly UM, Mitchell RG, Cook R, Steedman DJ, Robertson CE. Paramedics and technicians are equally successful at managing cardiac arrest outside hospital. BMJ 1995;310:1091-4.

2 Hussain LM, Redmond AD. Are pre-hospital deaths from accidental injury preventable? $B M J$ 1994;308:1077-80

3 Pelucio M, Halligan L, Dhindsa H. Out-of-hospital experience with the syringe esophageal detector device. Acad Emerg Med 1997;4:563-8.

4 Blostein PA, Koestner AJ, Hoak S. Failed rapid sequence intubation in trauma patients: Esophageal tracheal Combitube is a useful adjunct. J Trauma 1998;44:534-5.

5 Cooke MW. How much to do at the accident scene? BMJ 1999;319:1150. (Accepted 23 March 2001)

\section{Clarification}

Several readers reported to us an inconsistency between some illustrations in Eoin O'Brien and colleagues' ABC article on sphygmomanometry (21 April, pp 981-5) and what was said in a Clinical Review article by McAlister and Straus the previous week (14 April, pp 908-11). The ABC illustrations showed the diaphragm of the stethoscope being used, whereas McAlister and Straus, in their evidence based review of blood pressure measurement, said that the bell should be used (see "Guidelines" box in their article). The readers wondered which is the correct method (bell or diaphragm). It may come down to differences in practice between North America and the United Kingdom. O'Brien and colleagues told us that the British Hypertension Society's recommendations on blood pressure measurement advocate using the diaphragm rather than the bell, as no evidence exists to support using the bell in modern stethoscopes. McAlister and Straus said that evidence shows minimal or no difference between the bell and the diaphragm and that their article followed the guidelines of the American Society of Hypertension and the American Heart Association. See www.bmj.com/cgi/ eletters/322/7292/981 for the authors' full responses.

\author{
Department of \\ Accident and \\ Emergency, Royal \\ London Hospital, \\ London E1 1BB \\ David Lockey \\ fellow in prehospital \\ care \\ Gareth Davies \\ consultant in acciden \\ and emergency and \\ prehospital care \\ Tim Coats \\ senior lecturer \\ Correspondence to: \\ D Lockey \\ dj199@hotmail.com
}

BMJ 2001;323:141

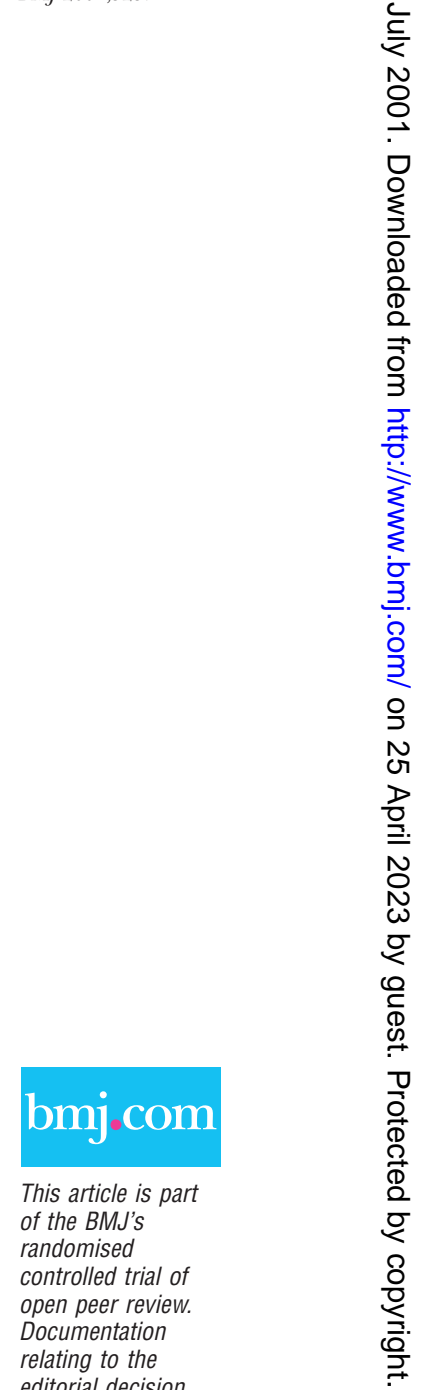

